\title{
Eutectic Synthesis of P2-Type
}

\section{$\mathrm{Na}_{\mathbf{x}} \mathrm{Fe}_{1 / 2} \mathrm{Mn}_{1 / 2} \mathrm{O}_{2}$ Cathode with Improved Cell Design for Sodium-Ion Batteries}

\author{
Mengya Li, ${ }^{1, *}$ David L. Wood, III, ${ }^{1,2}$ Yaocai Bai, ${ }^{1}$ Rachid Essehli, ${ }^{1}$ Ruhul Amin, ${ }^{1}$ \\ Charl Jafta, ${ }^{1}$ Nitin Muralidharan, ${ }^{1}$ Jianlin $\mathrm{Li}^{1,2}$ and Ilias Belharouak ${ }^{1,2, *}$ \\ ${ }^{(1)}$ Energy and Transportation Science Division, Oak Ridge National Laboratory, \\ Oak Ridge, TN 37830, USA \\ ${ }^{(2)}$ Bredesen Center for Interdisciplinary Research and Graduate Education, \\ University of Tennessee, Knoxville, TN 37996, USA
}

*Corresponding authors: Mengya Li (im1@ ornl.gov)

Ilias Belharouak (belharouaki@ornl.gov) 

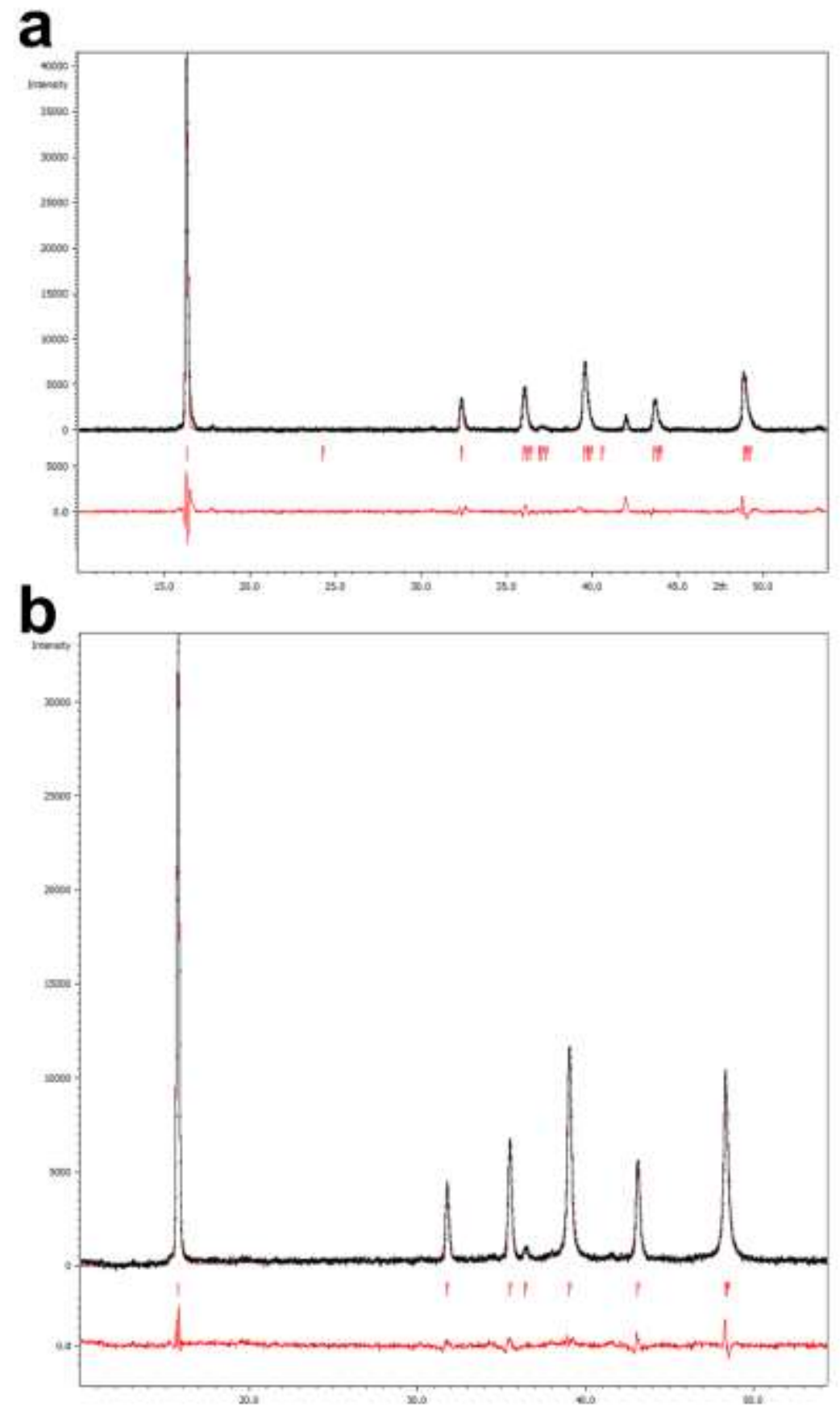

Figure S1. XRD profile matching refinement results for (a) SG-P2 and (b) EA-P2 $\mathrm{Na}_{\mathrm{x}} \mathrm{Fe}_{1 / 2} \mathrm{Mn}_{1 / 2} \mathrm{O}_{2}$. 

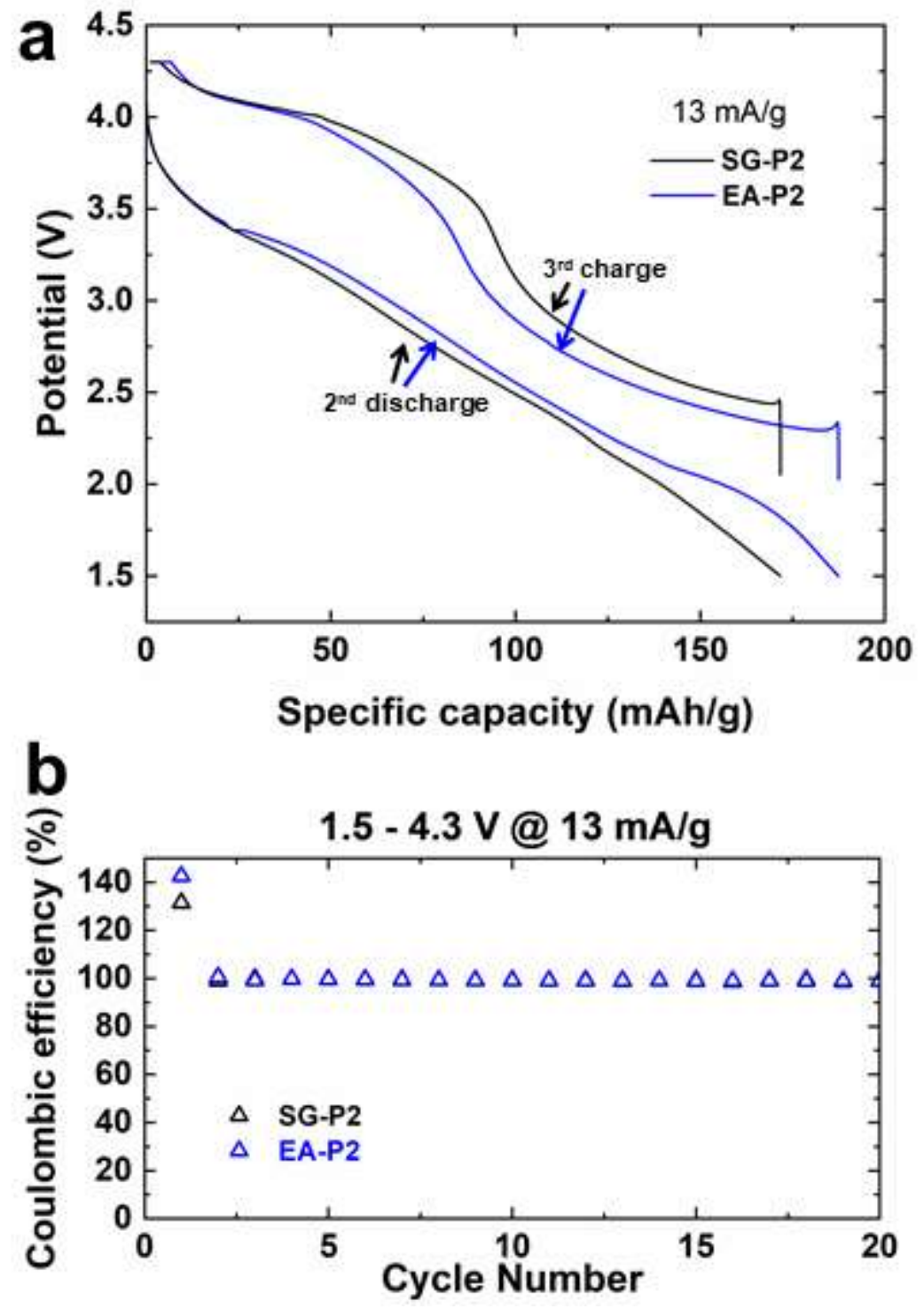

Figure S2. (a) Voltage curves of SG-P2 and EA-P2 $\mathrm{Na}_{x} \mathrm{Fe}_{1 / 2} \mathrm{Mn}_{1 / 2} \mathrm{O}_{2}$ in the $2^{\text {nd }}$ discharge and $3^{\text {rd }}$ charge. (b) Coulombic efficiency for SG-P2 and EA-P2 in SIB half cells upon cycling. 

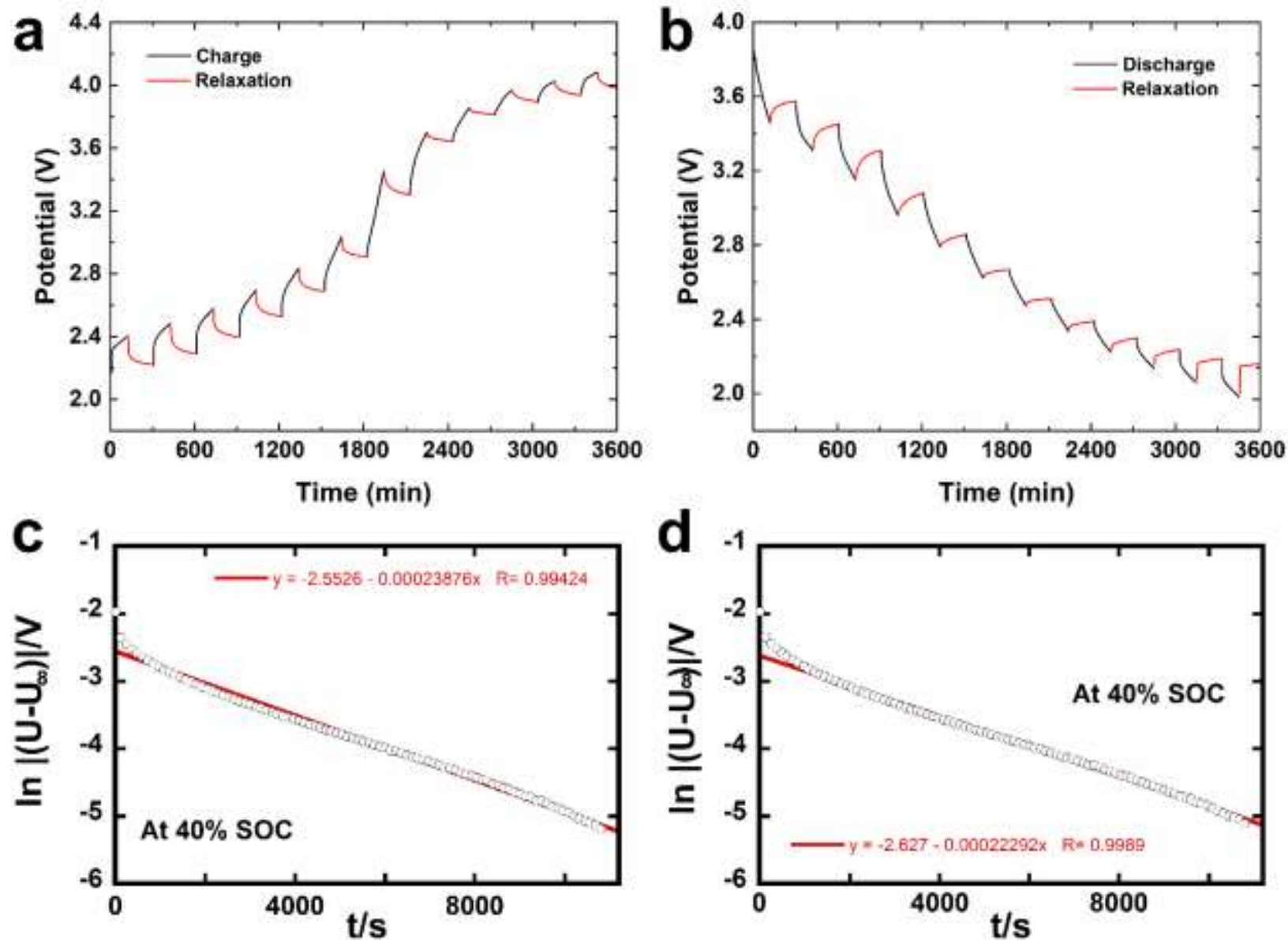

Figure S3. GITT (a) charge and (b) discharge profiles of EA-P2 $\mathrm{Na}_{x} \mathrm{Fe}_{1 / 2} \mathrm{Mn}_{1 / 2} \mathrm{O}_{2}$ cathode in SIB half cell. (c,d) Examples of fitting every relaxation period into a linear equation for deriving $\mathrm{Na}^{+}$ diffusion coefficient values. 

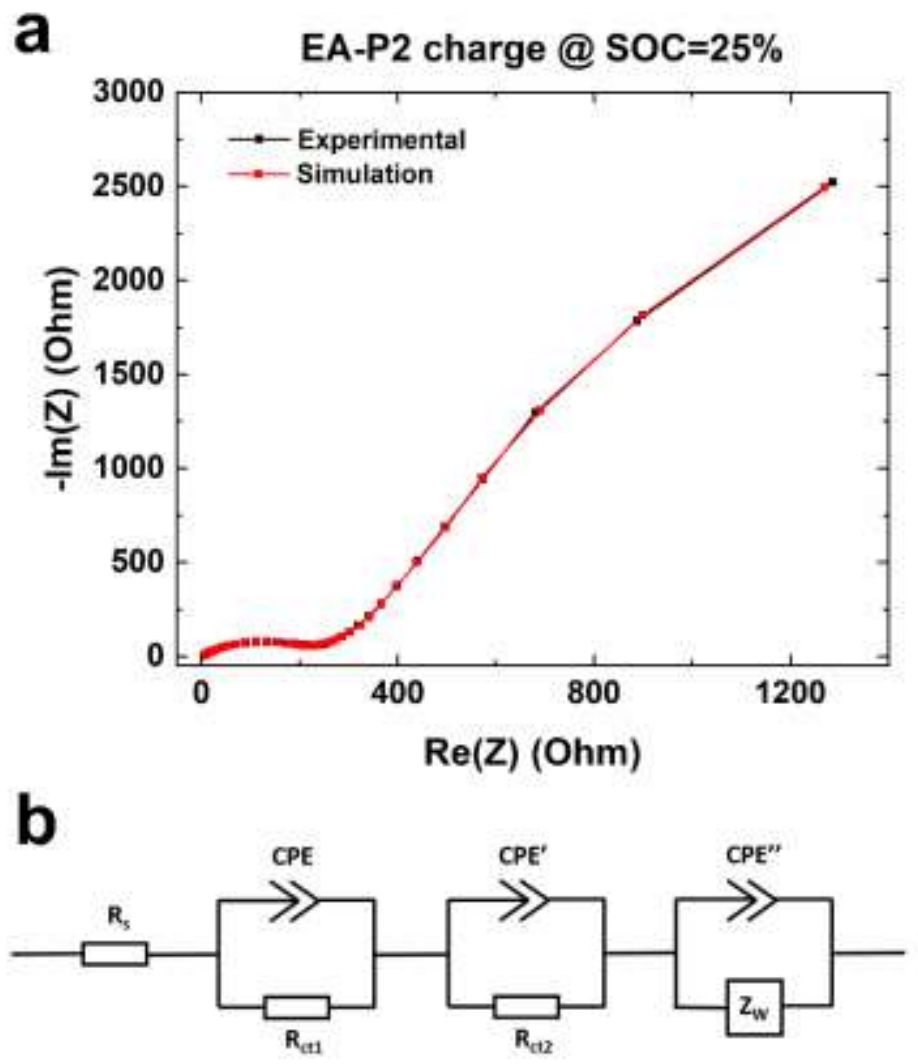

Figure S4. (a) Nyquist plot of EA-P2 cathode upon charging and corresponding EIS fitting results. (b) Equivalent circuit that was applied for the fitting of impedance spectra. 

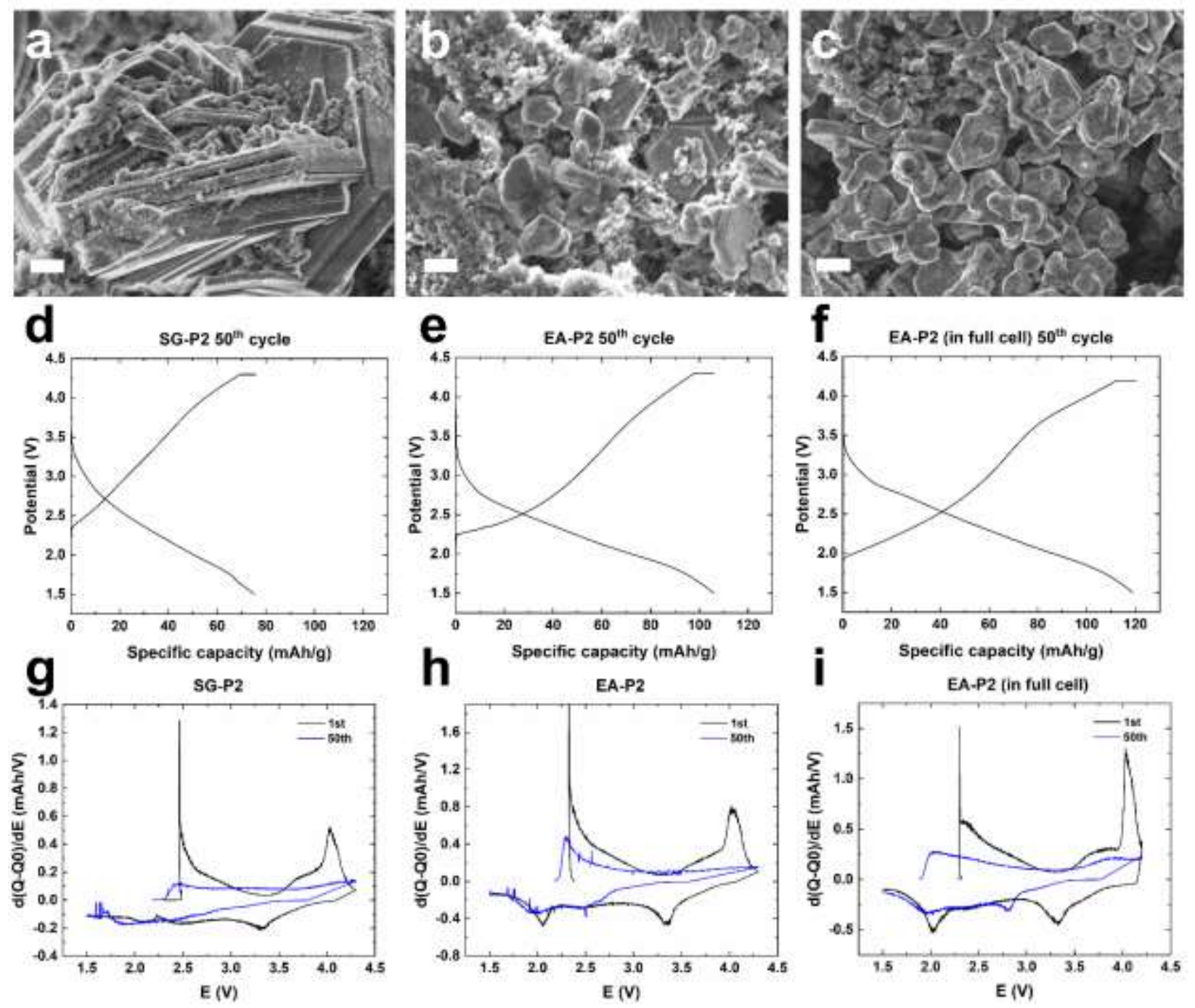

Figure S5. SEM of (a) SG-P2, (b) EA-P2, (c) EA-P2 (in full cell) $\mathrm{Na}_{x} \mathrm{Fe}_{1 / 2} \mathrm{Mn}_{1 / 2} \mathrm{O}_{2}$ cathodes after cycling test (all scale bars equal to $2 \mu \mathrm{m}$ ). Galvanostatic charge/discharge curves of the $50^{\text {th }}$ cycle for (d) SG-P2, (e) EA-P2, (f) EA-P2 (in full cell) SIBs. Corresponding dQ/dV profiles for the $1^{\text {st }} \& 50^{\text {th }}$ cycle of (g) SG-P2, (h) EA-P2, (i) EA-P2 (in full cell). 

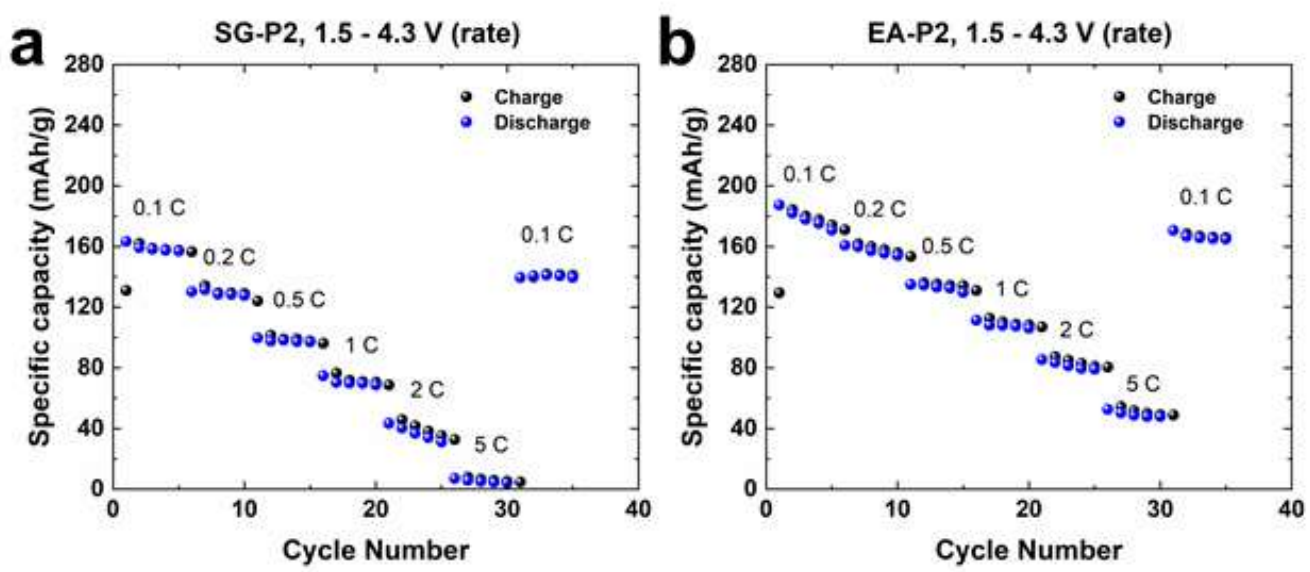

Figure S6. Rate-performance of SIB half cells with (a) SG-P2 and (b) EA-P2 $\mathrm{Na}_{x} \mathrm{Fe}_{1 / 2} \mathrm{Mn}_{1 / 2} \mathrm{O}_{2}$ cathodes. 


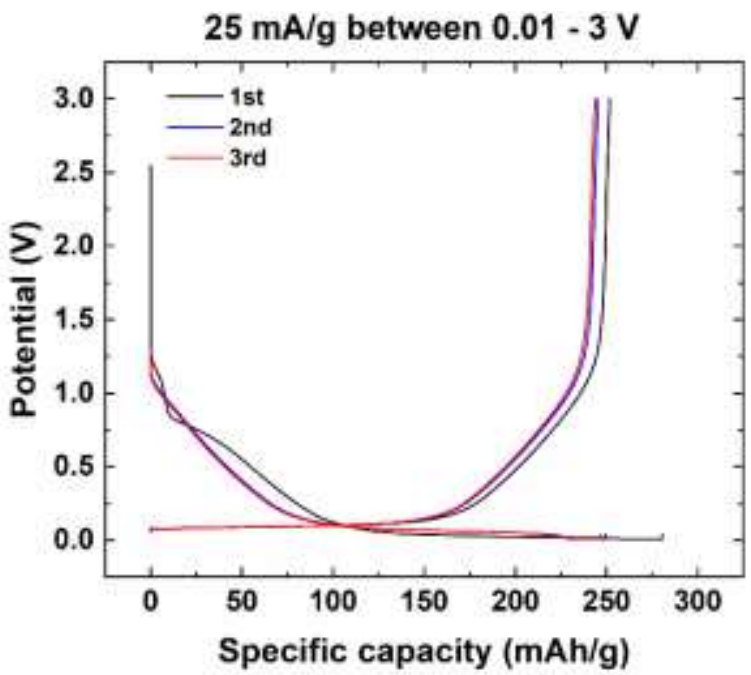

Figure S7. Galvanostatic charge/discharge profiles of a hard carbon anode against $\mathrm{Na}$ metal. 


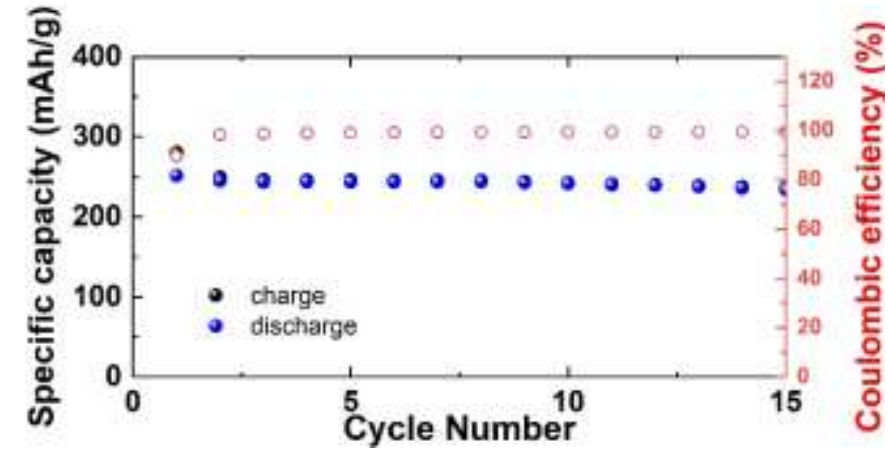

Figure S8. Cycling performance of hard carbon anode in SIB half cells $(0.1 \mathrm{C}$ between $0.01-$ $1.5 \mathrm{~V})$. 
Table S1. Mn/Fe atomic ratio obtained from SEM EDS results of different P2 materials after cycling.

\begin{tabular}{|c|c|c|c|}
\hline & SG-P2 & EA-P2 & EA-P2 (full cell) \\
\hline $\begin{array}{c}\text { Mn/Fe atomic } \\
\text { ratio (average) }\end{array}$ & 2.55 & 0.80 & 1.23 \\
\hline
\end{tabular}

